\title{
Infant lung function testing: available and useful methods
}

\section{Educational aims}

$>1$. To provide an overview of which aspects of lung function can be measured in infants using currently commercially available equipment.

$>2$. To describe the particular measurement conditions required for infant lung function testing.

$>3$. To provide information concerning some of the, as yet, commercially unavailable methods.

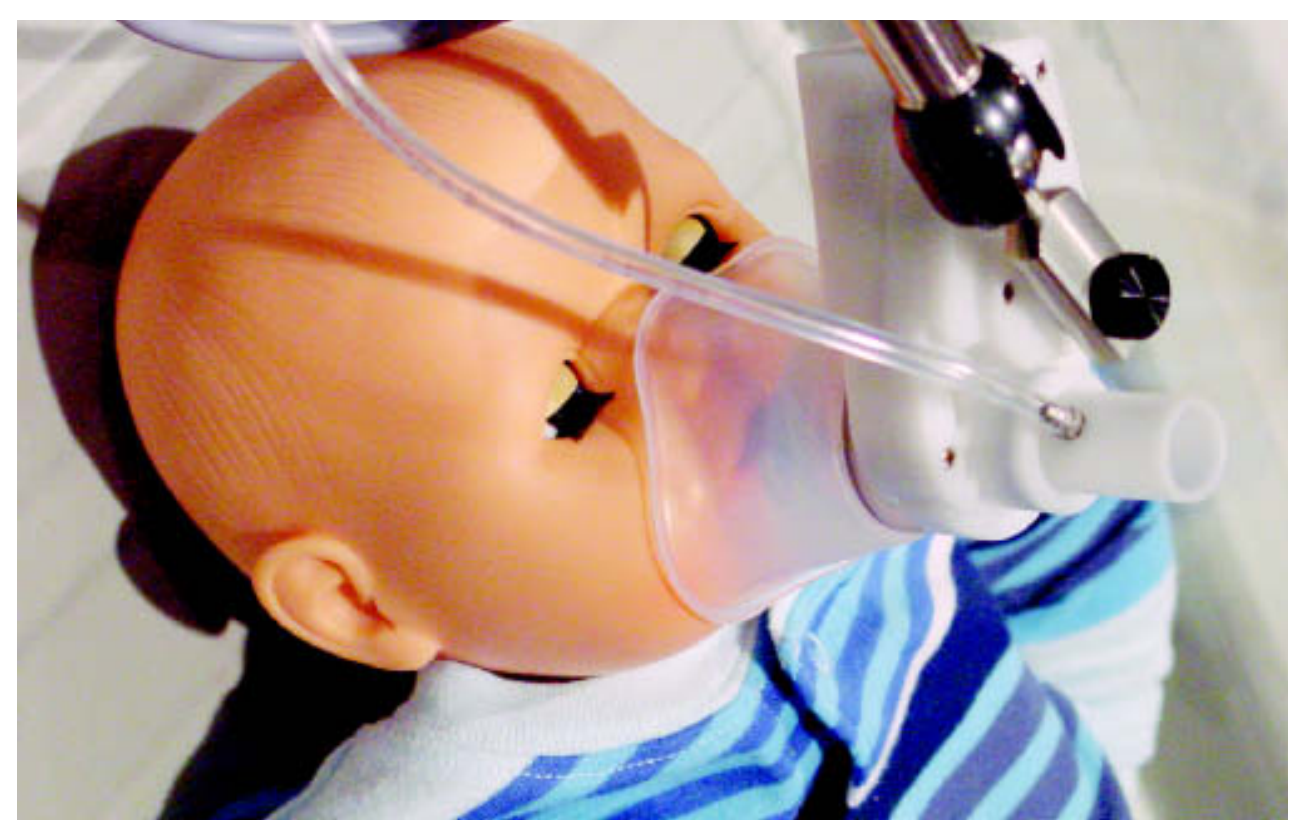

\section{Summary}

Lung and airway function can now be measured in infants using standardised methods, allowing for an almost as complete picture of lung function as routinely obtained in adults. Recording systems are now available from several manufacturers. The tests are done during sleep, commonly after sedation with choral hydrate, and in a strictly standardised manner under standardised conditions. Despite the high frequency of chronic chest problems in infants and young children, facilities for assessment of lung function are lacking in many large paediatric centres worldwide. While infant lung function testing is now emerging as a clinical tool, the precise role and the value of the various tests in different clinical situations remain to be established.

\section{H. Ljungberg \\ P.M. Gustafsson}

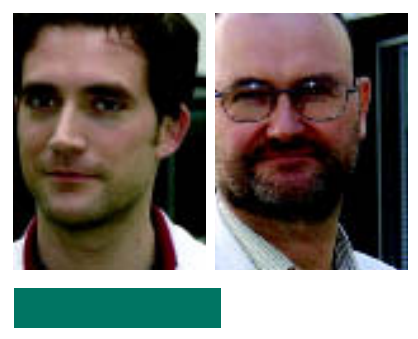

Dept of Paediatric Clinical

Physiology

Queen Silvia Children's Hospital Sahlgrenska University Hospital Göteborg

Sweden

\section{Correspondence:}

H. Ljungberg

Dept of Paediatric Clinical

Physiology

Queen Silvia Children's Hospital S-416 85 Göteborg

Sweden

Fax: 4631841605

E-mail:

henrik.ljungberg@pediat.gu.se

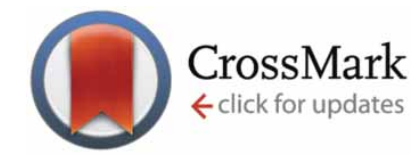




Keywords
< Many methods are now stan-
dardised and equipment has
recently become commercially
available
< ILFT may be used to provide
information about normal
growth and development of the
respiratory system; to detect,
classify and quantify lung dis-
ease; and to determine response
to interventions
$<$ The tests are time consuming
and require appropriate staff
training
$<$ Tests must be suited to/appro-
priate for the infant's suspected
condition
$<$ Care must be taken with newer
tests, as published reference
data may be inappropriate for
the specific equipment
$<$ Few studies addressing clinical
usefulness have been performed

\section{Figure 1}

The Infant Pulmonary Lab ${ }^{\circledR}$ marketed by Collins Medical Inc.

\section{Introduction}

【te The rationale for this review is emerging awareness of the usefulness of infant lung function testing (ILFT) in the diagnosis and management of infants with respiratory disorders. Its exact role, however, needs to be defined and the value of ILFT as a clinical tool needs to be established in clinical studies. Several manufacturers are now marketing advanced ILFT systems, and the clinician needs to be informed about their potential usefulness and their limitations.

Diseases of the airways are the single greatest cause of morbidity and mortality in infants and young children: intermittent problems with "wheezy chest" in the first years of life occur in more than a third of all children; asth$\mathrm{ma}$, the single most common chronic disease during childhood, starts very early in life in the majority of cases; cystic fibrosis (CF) is frequently diagnosed in infancy; and bronchopulmonary dysplasia (BPD), which is several times more common than $\mathrm{CF}$, is usually most severe during the first year of life. Despite the seriousness and significant costs of managing these conditions, the severity and character of the lung disease, and the effects of intervention

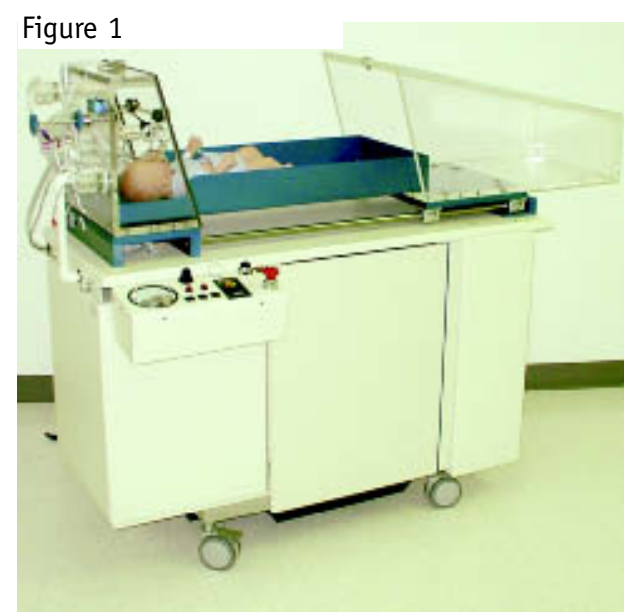

have been difficult to determine. Adequate assessment of the extent of the lung function reduction is currently not made in most cases, giving little opportunity to evaluate treatment on a large scale or in individual cases. Respiratory symptoms and signs, such as cough, wheezing, stridor, tachypnoea, hypoxaemia or chest retractions, are common in young children with various pathological conditions. They may have widely different causes and prognoses, and in many cases pose a challenge to the paediatrician. Is this a serious or chronic condition, or a trivial, benign and transient condition? What diagnostic procedures are most likely to give a rapid and correct diagnosis with the least risk for the child?

Radiographic methods, such as common chest radiography, helical and high-resolution computed tomography (CT) of the lungs, and fibreoptic bronchoscopy are wellestablished methods for clinical investigation in this age group, while physiological methods are used routinely only in a few centres. This contrasts with the management of school-aged children or adults with suspected or diagnosed lung disease, in whom lung function tests are used routinely as first-line investigations and when evaluating the course of a disease or the effect of any therapeutic interventions. However, it is now possible to measure lung function in infants and children below the age of 3 yrs using standardised commercially available equipment, and to obtain a picture of airway function almost as detailed as in a cooperative child or an adult. ILFT, however, demands special equipment, particular measurement conditions and procedures, and extensive personnel training, and it is far more time consuming than well-established methods for clinical investigation in infants [1].

Over the lastdecades, several ILFT methods have been developed and set up by researchers and paediatricians in different countries. These more or less homemade equipments were available for only a single or a few centres, and hardware, software and procedures were not standardised, making comparisons between findings in different laboratories more or less impossible. Early in the 1990s, a joint European Respiratory Society (ERS)/American Thoracic Society (ATS) working group, engaging physiologists and paediatricians from the USA, Europe and Australia, was organised with the intention to standardise methods and procedures with the techniques currently in use. This work was highly successful and has resulted in a handbook, which was published in 1996 [1], and several more recent publications [2-8], and the work is now being continued in the form of an ERS/ATS Task Force 
focusing mainly on lung function testing in preschool children.

The standardisation process has led to the marketing of almost complete labs for ILFT over the last few years from manufacturers such as: Collins Medical Inc. (fig. 1), Jaeger-Tonnies AG (fig. 2) and SensorMedics BV (fig. 3). These include tidal breathing analysis, passive lung mechanics, lung volume measurement using a body plethysmograph or an inert gas washout method, and forced expiratory volume and flow measurements. While these equipments can produce state-of-the-art measurements of lung function in infants, not all aspects of lung function can be quantified. For instance, there is no commercially available method to measure the diffusing capacity of the lungs.

At the time of birth, the human lung has acquired approximately half of its final number of alveoli, and alveolarisation is estimated to continue until about the age of 3 yrs. This means that the lung is in a state of rapid development during the first years of life, with a continually changing relationship between the size of the airways and the size and state of development of the gas exchange units. The result is markedly changing lung function in infants, followed longitudinally, and marked interindividual variability in different infants of a given age. During this period of rapid development, disease processes may affect lung structural development and lung function differently, depending on when the disease affects the lung most severely. Separating developmental differences from effects of disease on lung function in infants is, thus, a particular challenge when performing ILFT.

Previous ILFT studies have dealt with the following: describing the effects of different measurement conditions; comparisons of different techniques to measure a particular aspect of lung function; and age-related differences in particular parameters. Other studies have compared, on a group level, lung function as measured with a specific method in sick and healthy subjects [9]. While ILFT is not yet an evidence-based diagnostic procedure, the same can be said about lung function testing in other age groups. The major difference is that there is a great amount of clinical experience with lung function testing in older children and adults. However, the time has now come for studies
Figure 2

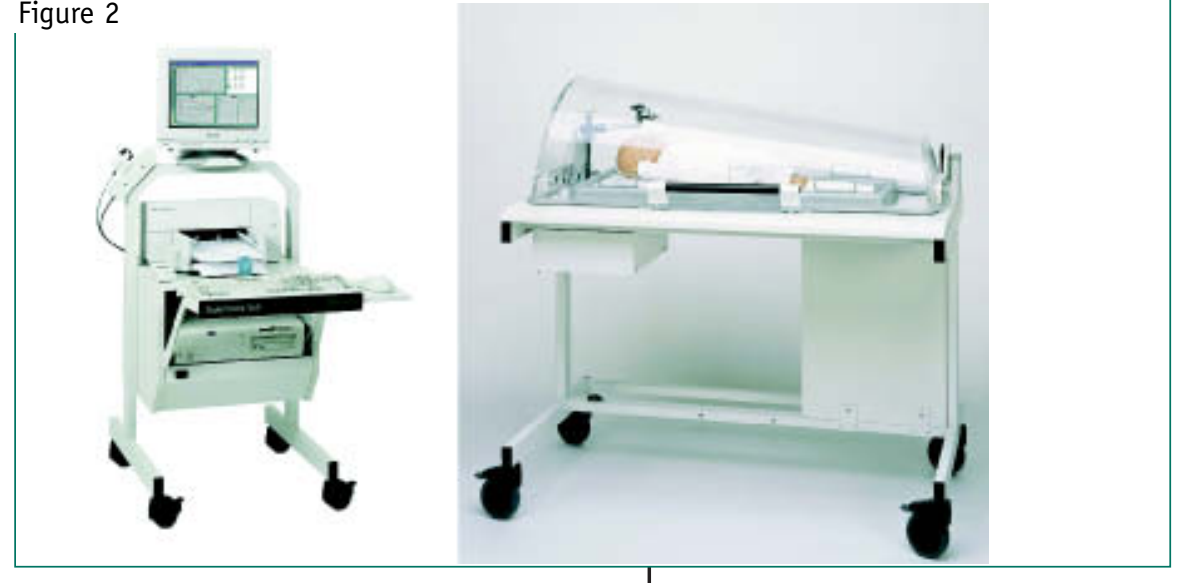

that will help clarify the role of ILFT as a routine clinical tool. Studies analysing which test is appropriate for a particular clinical situation are necessary, reporting the sensitivity and specificity of the test [9]. Normative data using the same method and equipment in a representative population of healthy subjects are highly desirable but not always available. The importance of using adequate reference values cannot be overemphasised.

Appropriate training of the personnel doing ILFT is of utmost importance. Some manufactures provide advanced training in their own facilities and on site in the buyer's laboratory. Basic understanding of ILFT is given at postgraduate courses given by the ERS and ATS, and a more advanced several-days-long course was arranged by the ERS in Davos, Switzerland, in April 2004.

In this paper, we will provide an overview of what aspects of lung function can be measured in infants using current commercially available equipment. The particular measurement conditions required for ILFT will be described. We will also report on some other not yet commercially available methods.

For further information on ERS school courses please go to: www.ersnet.org/school
Figure 3

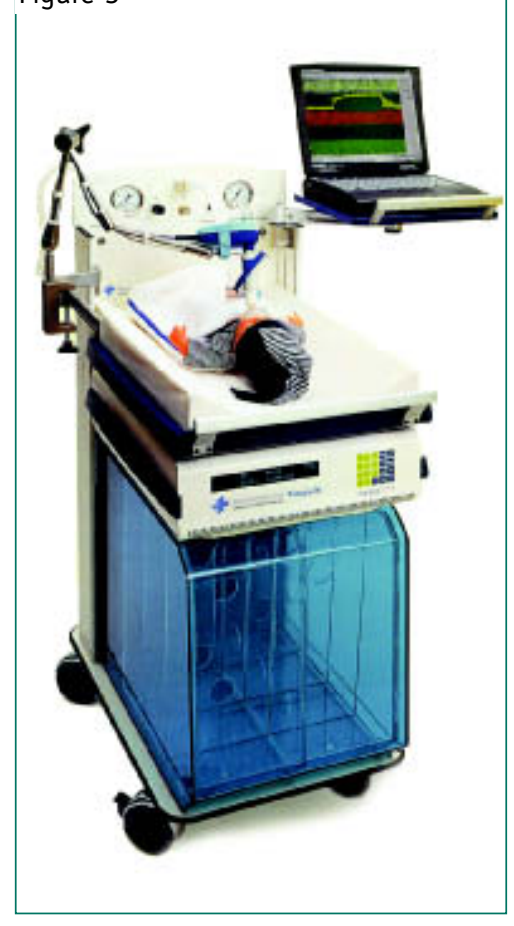

Figure 2

The Jaeger-Toennis Jaeger MasterScreen Paed ${ }^{\circledR}$ (left) and MS BabyBody ${ }^{\circledR}$ (right), marketed by Jaeger-Tonnies $A G$.

Figure 3

The Vmax $26^{\circledR}$ system marketed by SensorMedics BV. 


\section{Conditions required}

\section{Sedation or natural sleep?}

"An ideal ILFT should distinguish clearly between health and disease, be completely safe, acceptable to infants (and parents), simple to perform and reproducible."

\section{Figure 4}

Sedated sleeping infant with face mask and sealing putty. istered as an oral solution or rectally. be attained from relevant research ethics committees if collected data are to be used for research purposes and informed consent must be obtained from the parents.

\section{Contraindications}

Ongoing or recent upper or lower respiinfant lung function measurement are adapted from tests used in adults. Very young children are unable to actively cooperate or even remain passive during lung function measurement and, therefore, they need to be tested while asleep. In most instances, the use of a sedative is necessary, unless the infant is very young $(<1$ month). The most commonly used sedative is chloral hydrate [1], which only has very minor effects on respiratory pattern or breathing reflexes at the recommended dosages of 50-90 mg.kg body weight ${ }^{1}$. It may be admin-

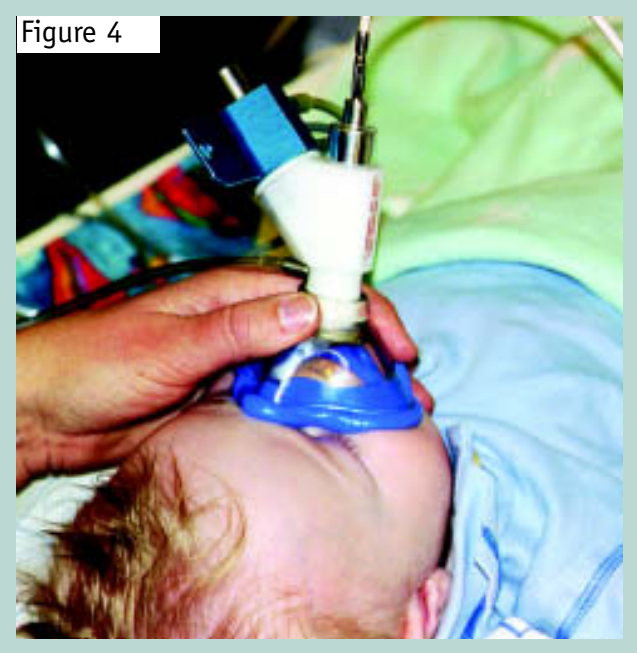

\section{Preparation and parental information}

Parents must be provided with adequate information about the procedures and sedation with chloral hydrate. For this purpose, a prepared leaflet with explanatory photographs as well as written information about the procedures is useful. Infants do not need to be fasting before sedation with chloral hydrate and a small feed before testing helps improve sleep induction, which is generally achieved within 20-30 minutes. Before administration, the child's weight, length and oxygen saturation (pulse oximetry) are measured. Information about the child's general and respiratory health is collected through parental interview, and a physician examines the infant. Approval must ratory tract infections (RTI) constitute contraindications to IFLT. Ideally, the infant should be free of symptoms from the RTI for 2 weeks before the investigation. An upper RTI may interfere with measurements by affecting the lower airways, e.g. due to post-nasal drip, but, more importantly, even mild nasal obstruction may have a marked effect on airway resistance. Great caution must be observed when sedating infants with a history suggesting the presence of upper airway obstruction. In such infants, sedation may elicit acute obstruction, precluding further assessments of lung function. Caution is also recommended with neurologically handicapped children, who may also develop upper airway obstruction when sedated. In many of these infants, a sleep study prior to ILFT may be useful to assess the risks involved. Correctly undertaken, the risk for a serious adverse event requiring resuscitation is very small. We have investigated well over 1,000 infants and have never been faced with such a situation. Similar experience is reported from the laboratories we have regular contact with in different countries.

\section{Test procedure}

Suggested requirements for equipment, test conditions and personnel training have been described previously [1]. A minimum of two people (suitably medical doctors or registered nurses) should perform the tests together. They must have adequate training, and need to have acquired the necessary experience and familiarity with regards to equipment, sedation and monitoring of infants, as well as airway management. Measurement of lung function is generally performed with the infant in the supine position, with the head and neck slightly extended, during episodes of "quiet sleep" [1]. This is usually defined by a regular, quiet breathing pattern, and by absence of movement of the eyes or extremities. A mask constructed from a transparent, stiff material is placed over the infant's mouth and nose. A ring of silicone putty 
is generally used to seal off any air leaks. The mask can then be connected to the relevant measurement device, e.g. a flow meter and a pressure transducer (fig. 4). The equipment used should be suitable for the infant size, with appropriate instrumental dead space and resistance, be safe and adhere to published guidelines [1-7]. The equipment must be maintained and regularly calibrated according to the manufacturer's instructions. ILFT should be performed in an environment with suitable lighting, noise level and temperature, and acceptable in terms of infection control [1].
During testing, infants should be continuously monitored using pulse oximetry. Resuscitation equipment, including oxygen supply and suction apparatus, must be available. All testing, including the infant's status, should continually be adequately documented. Data storage regulations must be adhered to.

\section{Overview of methods}

An ideal test should distinguish clearly between health and disease, and it should be completely safe, acceptable to infants (and parents), simple to perform and reproducible. These requirements are clearly not yet fulfilled for any tests available. What test should then be used? Obviously this should not depend on which test modules have been bought to the lab but on the clinical question at hand. What characterises the condition of the infant with suspected lung disease, and which methods may be most suitable to diagnose and assess the severity of that condition? In many cases there may not be a "single best test" but a combination of tests may be the best approach. The availability of appropriate reference data may also influence the choice of method.

The methods most commonly used and some promising new tests (table 1) are reviewed below. While most of the tests have been developed over the last decades, some have become commercially available only very recently. Table 2 shows which tests are available from the marketed recording systems. The recommendations with regards to standard of hardware, measurement procedures and evaluation have been published for most techniques [1-7]. It is emphasised that the methods reflect different aspects of the respiratory system. The various tests are appropriate for different purposes, in different conditions and settings, as discussed separately for each technique.

\section{Table 1. - Overview of methods available for testing lung function in sedated infants $<3$ yrs \\ $V_{T}$ and, RR, flow-time and flow-volume relationships $C_{r s}, R_{r s}$ and $T_{r s}$ Partial forced expiratory flow/volume loops, flow at functional residual capacity ( $V_{\text {max, }}^{\prime}$,RC) Maximal expiratory flow/volume loops: FVC, FEV 0.4 , FEV $0.5, M E F_{50}$, MEF 25 , etc. \\ FRC and Raw \\ FRC and indices of ventilation maldistribution Airway and parenchymal mechanics.}

TRTC: tidal rapid thoracic compression technique; RVRTC: raised volume rapid thoracic compression technique; FOT: forced oscillation technique; VT: tidal volume; RR: respiratory rate; $C_{r s}$ : compliance of the respiratory system; Rrs: resistance of the respiratory system; Trs: expiratory time constant of the respiratory system; FRC: functional residual capacity; Raw: airways resistance.

Table 2. - Commercially available systems for measuring infant lung function

\section{Collins Medical Infant Pulmonary} $L a b^{\circledR}$

Measurement of tidal breathing, passive mechanics, plethysmography, airway resistance, tidal rapid thoracic compression technique and raised volume rapid thoracic compression technique

\section{Jaeger MasterScreen Paed/BabyBody ${ }^{\circledR}$}

Measurement of tidal breathing, passive mechanics, plethysmography, airway resistance and tidal rapid thoracic compression technique; raised volume rapid thoracic compression technique soon available

\section{Sensormedics Vmax $26^{\circledR}$}

Measurement of tidal breathing, passive mechanics, tidal rapid thoracic compression technique, raised volume rapid thoracic compression technique, and functional residual capacity and ventilation distribution parameters measured by multiple-breath washout; exhaled nitric oxide also available 


\section{Figure 5}

a) Volume-time and b) flow-time traces recorded during tidal volume (VT). PTIF: peak tidal inspiratory flow; PTEF: peak tidal expiratory flow; TI: inspiratory time; TE: expiratory time; TTOT: total time; TPTIF: peak tidal inspiratory flow time; TPTEF: peak tidal expiratory flow time.

\section{Figure 6}

Expiratory and inspiratory flow/volume loop recorded during tidal volume (VT). VPTEF: volume at peak tidal expiratory flow; PTEF: peak tidal expiratory flow; PTIF: peak tidal inspiratory flow.

\section{Figure 7}

Measurement of passive respiratory system mechanics. Flow/volume loop recorded during passive lung deflation after eliciting the Hering-Breuer inspiratory reflex. The interval from $A$ to $B$ indicates the extrapolated tidal volume, and $C$ to $D$ indicates the linear portion of the deflation curve from which the time constant of the respiratory system is measured.

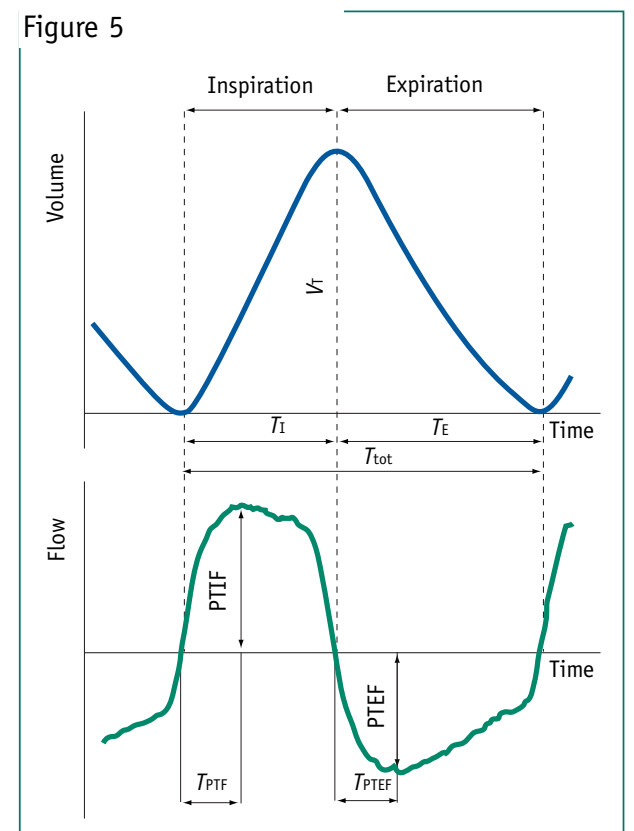

Figure 6

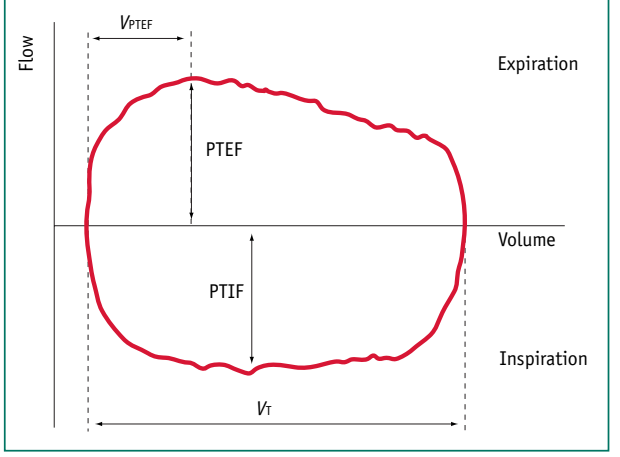

Figure 7

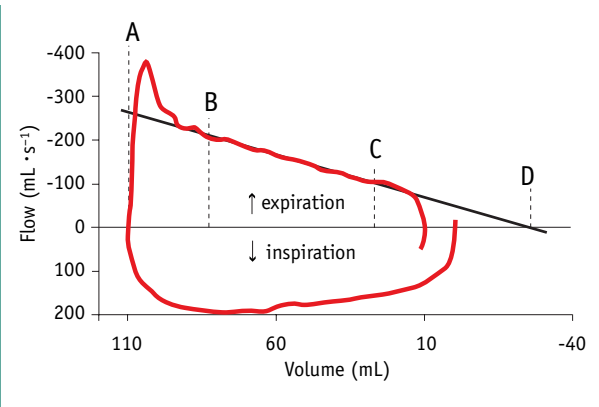

\section{Tidal breathing} parameters

This is a relatively simple, noninvasive technique to determine the breathing pattern. Expiratory and inspiratory flow and volume are recorded using a flow meter. Flow-time, volumetime (fig. 5) and flow-volume (fig. 6) tracings are generally displayed online. Tidal volume $\left(V_{T}\right)$, respiratory rate (RR), time to peak expiratory flow (as percentage of total expiratory time) and the shape of the flow/volume loops are commonly reported. The data will reflect a mixture of lung mechanics and control of breathing. It is also a useful measure to help confirm that the child is in quiet sleep. It may be possible to determine if an upper airway obstruction is present, and help determine whether this is extrathoracic or intrathoracic. Whereas the presence of severe bronchial obstruction is usually visible from the recordings of tidal breathing, the diagnostic sensitivity and specificity for airway obstruction is limited.

\section{Passive mechanics of the respiratory system}

This method can be used to determine the compliance $\left(C_{\mathrm{rs}}\right)$ and resistance $\left(R_{\mathrm{rs}}\right)$ of the respiratory system. The respiratory system includes the chest wall and airways, as well as the lung parenchyma. Under 18 months of age, however, the chest wall is very compliant and therefore the $C_{\text {rs }}$ will mainly reflect the lung compliance. Technically the single occlusion technique is most commonly used and involves a brief end-inspiratory occlusion of the airways to invoke the Hering-Breuer reflex. This reflex causes the glottis to open and the respiratory musculature to relax and a totally passive expiration to occur (fig. 7). The $C_{\text {rs }}$ is calculated as the ratio of the extrapolated $V T$ and airway pressure at relaxation after occlusion. The time constant of the respiratory system $\left(T_{\mathrm{rs}}\right)$ is calculated from the slope of the linear portion of the passive expiratory flow-volume curve. The $R_{\mathrm{rs}}$ can then be calculated from the relationship: $T_{\text {rs }}=R_{r s} \times C_{r s} . C_{r s}$ results may be expressed in relation to body weight or in relation to the functional residual capacity (FRC), and is then known as the "specific compliance". This technique may be used to confirm restrictive pulmonary changes (such as may occur early in BPD or in interstitial lung diseases), but is of limited value in individual patients. The Rrs is heavily influenced by upper airway patency, and severe obstruction may result in a nonlinear expiratory portion of the flow-volume curve. These properties make the test a relatively blunt measure of bronchial obstruction, and often invalidate calculations in cases of severe obstruction. 


\section{Forced expiratory flow/volume loops}

Measuring forced expiratory flow/volume loops allows for determination of the severity of bronchial obstruction. The maximal flow that can be achieved at a given lung volume depends on the airway and parenchymal mechanical properties, and the technique is used to measure the maximal flow at a given lung volume. Once a certain minimum effort has been exceeded, maximum expiratory flow becomes independent of the effort applied. In a sleeping or sedated infant, an inflatable bladder is placed over the chest and abdomen. A non-elastic vest (fig. 8) is wrapped around the infant, and a bladder is inflated at end-expiration, causing the infant to breath out. Flow limitation is achieved by gradually increasing the pressure used to inflate the bladder.

There are two methods to record forced expiratory flows in infants: the tidal rapid thoracic compression (TRTC) technique and the raised volume rapid thoracic compression (RVRTC) technique [1]

\section{The TRTC method ("tidal squeeze")}

Measurement of partial forced expiratory flow/volume loops using the TRTC technique was established first, and is performed by inflating the bladder after a normal tidal inspiration during quiet breathing. The maximal flow at FRC, i.e. at the spontaneous end-expiratory level, is recorded. The pressure used to inflate the bladder normally starts $\sim 3$ $\mathrm{kPa}\left(\sim 23 \mathrm{cmH}_{2} \mathrm{O}\right)$, and is increased in steps of $1 \mathrm{kPa}$ until a maximal flow value is reached. This is termed the $V_{\text {max,FRC (fig. 9). }}$

While reference values that have been established for this technique [10] are available to download as a macro from the web (see useful web links table), interpretation of recorded data is complicated by the fact that there is a very wide range of what can be considered a normal $V_{\text {max,FRC }}$ in health. The technique also has potential for performing reversibility testing using an inhaled $\beta_{2}$-agonist [11]. However, as the FRC is dynamically maintained in infancy, this also makes interpretation of the data more difficult, as any change in FRC will shift the flowvolume curve (fig. 9) and confound results. FRC alterations may occur in health with a change

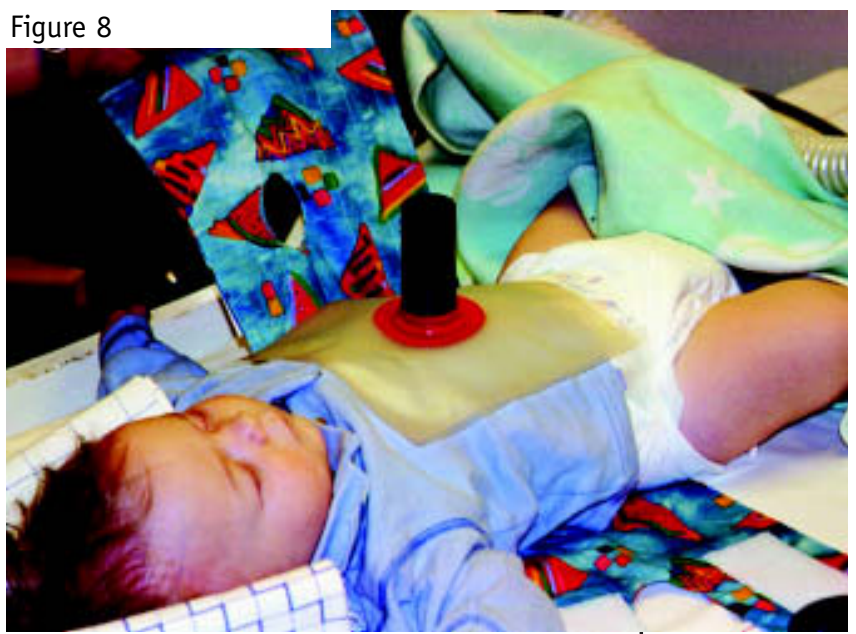

in instrumental dead space, with changes in sleep state or breathing pattern, or secondary to disease, therapeutic interventions or bronchial challenges.

\section{The RVRTC method ("raised volume squeeze")}

By extending the volume range, maximal forced expiratory flow/volume loops, similar to spirometric measurements in older children and adults, may be achieved. A pressure of $2.6-4.0 \mathrm{kPa}\left(20-30 \mathrm{CmH}_{2} \mathrm{O}\right)$ [1, 12-14] is then applied to the airway opening, elevating the infant's lung volume passively to near total lung capacity. When the positive pressure is removed, the infant exhales spontaneously, and this is repeated 4-5 times. As the last passive inflation reaches maximal inspired volume, the bladder is inflated and a maximal expiratory flow-volume curve loop is registered (fig. 10). This manoeuvre allows for estimation of the forced vital capacity (FVC) (from $4.0 \mathrm{kPa}\left(30 \mathrm{cmH}_{2} \mathrm{O}\right)$ ), timed forced
Figure 9

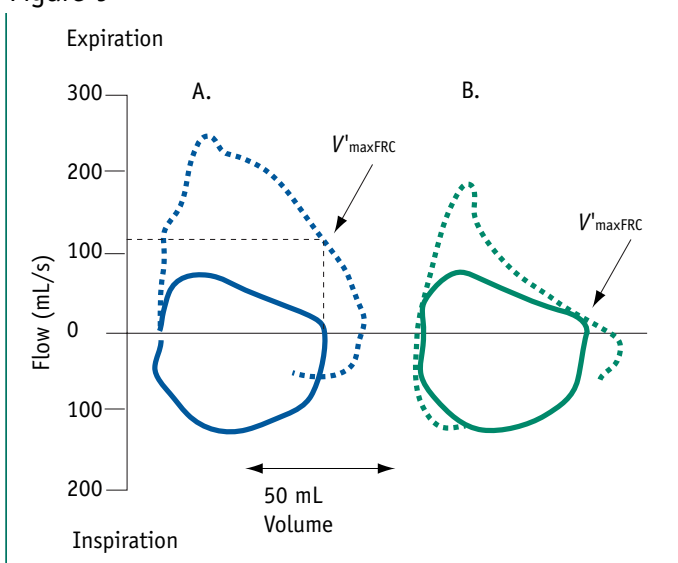

Figure 8

Pressure bladder and vest used to achieve forced expiratory flows and volumes in infancy.

\section{Figure 9}

Partial forced expiratory flow/volume curves (----) in a) a healthy infant and in b) an infant with severe airway obstruction and flow limitation. The preceding tidal breath is shown 


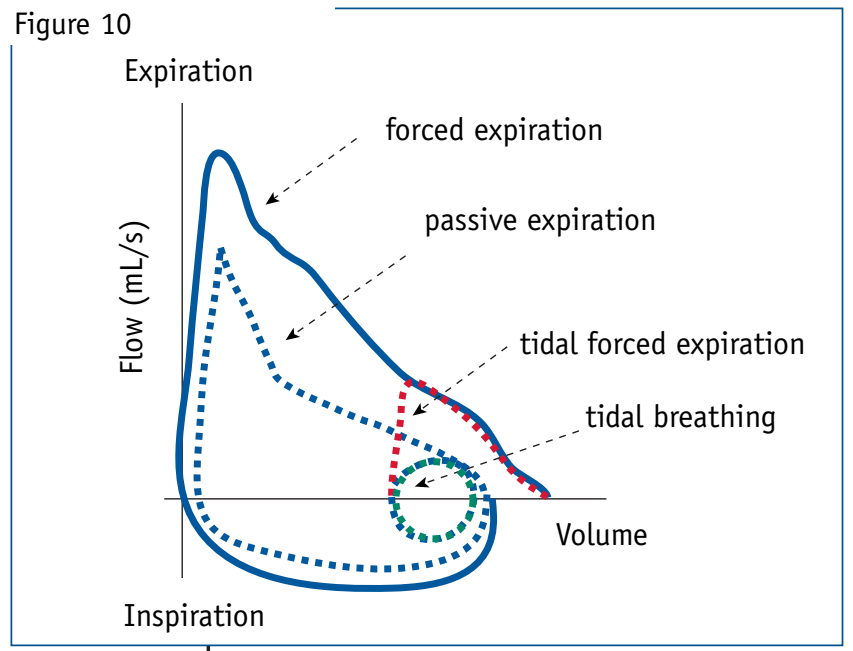

\section{Figure 10}

Maximal forced expiratory flow/volume loop (--) in an infant and preceding passive expiration after passive inspiration to an airway pressure of 3.0 kPa. A partial forced expiratory flow/volume loop measured shortly before in the same patient is shown (----). The partial (tidal forced expiration) and maximal curves overlie, which is to be expected as flow limitation has been reached. The tidal breathing loops are also presented. expiratory volumes, usually at 0.4 or $0.5 \mathrm{~s}$ and forced expiratory flows at different lung volumes, i.e. very similar measures to those recorded during conventional spirometry. Although this technique is very promising, as it avoids many of the drawbacks of the tidal rapid abdominal compression technique, and facilitates interpretation and longitudinal evaluation of data, there are some methodological issues to be resolved and, as yet, no reference values are available. Nevertheless, the technique is widely used and is appealing, as parameters recorded are similar to those recorded during spirometry in older children and adults. In addition, the reduced variability compared to $V_{\text {max, FRC }}$ makes longitudinal measurements, reversibility testing or bronchial challenges more feasible, and the RVRTC technique has been shown to be a sensitive measure of early lung changes in infants with CF [15].

\section{Functional residual capacity measurements}

The FRC is the volume of gas that remains in the lungs after a spontaneous exhalation, and is the only static lung volume currently measured routinely. It may be measured using infant whole body plethysmography or by gas dilution techniques. A correct measure of FRC is of great value when interpreting volume-dependent measures, such as lung mechanics and forced expiratory flow data ( $V$ max, FRC).

\section{Plethysmographic FRC}

Commercially available infant plethysmographs (figs 1 and 2), although bulky and expensive, have improved the ease of use, and allow for rapid and reliable determination of FRC. While in quiet sleep in the plethysmograph and with a stable end-expiratory level, the infant makes respiratory efforts against a closed shutter for a few seconds. Pressure $(P)$ and volume $(V)$ are recorded within the plethysmograph. By use of Boyle's law ( $P \times V=$ constant), the infant's lung volume may be calculated from the relationship: FRC $\approx(\triangle V / \triangle P) \times P_{B}$, where $\Delta V$ reflects changes in plethysmograph volume, $\triangle$ Preflects changes in airway pressure during respiratory efforts and $P_{\mathrm{B}}$ is the ambient barometric pressure.

This measure is of clinical importance, as it can be used to detect hyperinflation, as may occur with intrathoracic airway obstruction (obstructive disorders), and to indicate restrictive lung disorders including hypoplasia. As a result of improved measurement accuracy achievable with new equipment, there has been a gradual reduction in reported plethysmographic FRC values from $\sim 35$ to $20 \mathrm{~mL} \cdot \mathrm{kg}^{-1}$ in healthy infants during the last 30 yrs [16]. Therefore, until normative data appropriate for the new systems are published, values must be interpreted with caution. As plethysmography measures all compressible intrathoracic gas, it is a more useful measure of hyperinflation than gas dilution techniques. The latter may underestimate hyperinflation, as only volumes communicating with the central airways during tidal breathing will be included. The two techniques may, therefore, yield different results in obstructive subjects. A significantly higher plethysmographic than gas dilution FRC indicates the presence of trapped gas and, indirectly, peripheral airway obstruction. Plethysmography may, however, in severe cases of airway obstruction, overestimate lung volume.

Infant plethysmography also allows for determination of the airways resistance (Raw) and the specific airway resistance (sRaw). Raw probably reflects central airway obstruction, but the exact clinical value of this parameter still needs to be evaluated further.

\section{FRC measured by gas dilution}

Measurement of FRC using inert tracer gases can be performed using either the closed circuitdilution technique [17] or an open circuit 
multiple-breath washout(MBW) technique [18, 19]. Both measure the lung volumes (FRC) that readily communicate with the airway opening during tidal breathing by use of an inert tracer gas such as $\mathrm{He}_{1} \mathrm{~N}_{2}$ or $\mathrm{SF}_{6}$. These inert gases are not significantly absorbed into the body or emitted to the lungs from body tissue during the time of a test, and do not participate in gas exchange. The closed circuit He dilution test is not widely used, as it may only be used to calculate the FRC. In contrast, there is an increasing awareness of the potential diagnostic value of the inert gas MBW technique, which not only measures the FRC, but also provides various indices of uneven ventilation distribution if flow and gas concentration are measured simultaneously [19]. By measuring the homogeneity of ventilation distribution, even minor peripheral airway obstruction, difficult to detect using other techniques, may be measured [20]. The MBW test is performed using washout of $\mathrm{N}_{2}$, using $100 \% \mathrm{O}_{2}$ or washout of other inert gases ( $\left.\mathrm{He}_{\mathrm{r}} \mathrm{SF}_{6}\right)$ using room air after a small amount of the inert gas has been equilibrated into the lungs. FRC is calculated as the ratio between cumulative expired tracer gas volume and the difference in end-tidal tracer gas concentration at the start and the end of the washout [19].

Clinically, while FRC measured using gas dilution may be less useful for detecting hyperinflation than plethysmographic FRC, assessments of ventilation maldistribution may be important when evaluating suspected peripheral airway disorders, such as early CF lung disease and obliterative bronchiolitis. Presently, only one manufacturer (Sensor Medics BV) has marketed equipment for assessment of FRC and ventilation distribution.

\section{Forced oscillation technique}

The forced oscillation technique (FOT) may be used in infants [21] to estimate airway and parenchymal mechanical properties. Measurements are performed during an induced Hering-Breuer reflex using low-frequency forced oscillation, and the airway and parenchymal contribution to resistance is calculated. While standardisation of methodology still remains for this technique, it has been used to measure bronchodilator and methacholine responses, particularly in preschool children, as well as in epidemiological studies. There is no commercial FOT system available for use in infants.

\section{Diffusing capacity of the lung}

It is not currently possible to determine the diffusing capacity in infants using commercially available systems. Such a method would allow for assessment of the alveolar area available for gas exchange and hence indirectly the number of functioning alveoli. It would be very useful to measure the extent to which lungs of prematurely born infants are developed, and to evaluate any effects of therapeutic interventions in such patients and in those with bronchopulmonary dysplasia.

\section{Other methods}

Lung function tests performed at neonatal or paediatric intensive care units are beyond the scope of this paper. Monitoring of chest and abdominal movements by respiratory inductance plethysmography has been used in many research studies but appears to have little place in the diagnostic work-up of infants with suspected chest disease. Transcutaneous measurement of blood gases may also have a role when monitoring infants with severe respiratory disorders but is not the focus of this paper.

\section{Role of ILFT in clinical evaluation of infants with respiratory disorders}

The most common reason why infants have undergone lung function measurementso far has been participation in research studies [9]. The clinical value or usefulness of adding ILFT to the traditional diagnostic set-up, such as chest radiography, $\mathrm{CT}$ of the lungs and bronchoscopy, is yet to be established, and there is a lack of scientific studies addressing which particular ILFT is most helpful when evaluating infants with specific symptoms or known respiratory disorders. Many researchers have used available equipment rather than the ILFT methods that are expected to be the most appropriate when evaluating lung function impairment in infants with different conditions or when assessing the effect of interventions. For example, the TRTC test $\left(V_{\text {max }}\right.$,FC $)$ may not be the most suitable for determining the response
"There is no single universal test allowing diagnosis, assessment of severity, and the therapeutic response of an infant presenting with signs and symptoms of a respiratory disorder." 


\section{Suggested further reading}

$>1$. The comprehensive guide to procedures and analysis of infant lung function testing.

Stocks J, Sly PD, Tepper RS, Morgan WJ, eds. Infant Respiratory Function Testing. New York, John Wiley \& Sons, Inc, 1996. Contains theoretical background, test descriptions, as well as practical aspects and troubleshooting sections.

\section{>2. Godfrey S, Bar-Yishay, Avital A,} Springer $C$.

What is the role of tests of lung function in the management of infants with lung disease?

Pediatr Pulmonol 2003; 36: 1-9. $A$ recent review of literature and the current role of infant lung function testing worldwide.

> 3. Stocks J, Sly PD, Morris MG, Frey U. Standards for infant respiratory function testing: what(ever) next?

Eur Respir J 2000; 16: 581-584. The editorial to the joint ERS/ATS taskforce for Standards for Infant Respiratory Function Testing. to bronchodilator therapy. As mentioned previously, bronchodilation may reduce hyperinflation and cause a reduction of the absolute lung volume at which $V_{\text {max,FRC is meas- }}$ ured. Despite a widening of the airways, one may, therefore, observe a reduced $V$ max, FRC value (fig. 9) despite a positive response to the agent! There is, unfortunately, no single universal test allowing diagnosis, assessment of severity, and the therapeutic response of an infant presenting with signs and symptoms of a respiratory disorder, such as tachypnoea, wheezing, cough, chest deformity or failure to thrive. A number of techniques used in combination may be required to arrive at a plausible explanation of the infant's respiratory problems.

The role and use of ILFT have to be put into perspective, whilst, for example, an obstructive disorder may be quite well characterised in terms of location and severity, and the response to therapeutic intervention may be assessed, it goes without saying that the aetiology of the condition cannot be determined using ILFT. It is, however, our experience that IFLT can provide valuable additional information in cases where the diagnostic issues are complicated. For instance, it may be possible to establish to what extent the infant's symptoms originate from the respiratory system, and other causes, such as cardiac problems, most likely can be excluded, and vice versa. Importantly, ILFT provides the opportunity for objective assessment of therapeutic intervention. In chronic lung diseases where early intervention may be of particular importance for long-term outcome, such as cystic fibrosis, IFLT may come to play a very important role.

\section{Educational questions (Answers on page 23)}

1. Which methods can be used for lung volume (FRC) assessment in infants?

2. In what respect does the information given by these tests differ?

3. What is the rationale for using the raised volume rapid thoracic compression (RVRTC) instead of the tidal thoracic compression (TRTC) test?

4. Which ILFT methods provide information on peripheral airway function?

\section{References}

1. Stocks J, Sly PD, Tepper RS, Morgan WJ. Infant respiratory function testing. New York, Wiley-Liss, 1996.

2. Morris MG, Gustafsson P, Tepper R, Gappa M, Stocks J. The bias flow nitrogen washout technique for measuring the functional residual capacity in infants. ERS/ATS Task Force on Standards for Infant Respiratory Function Testing. Eur Respir J 2001; 17: 529-536.

3. Stocks J, Godfrey S, Beardsmore C, Bar-Yishay E, Castile R. Plethysmographic measurements of lung volume and airway resistance. ERS/ATS Task Force on Standards for Infant Respiratory Function Testing. European Respiratory Society/American Thoracic Society. Eur Respir J 2001; 17: 302-312.

4. Bates JH, Schmalisch G, Filbrun D, Stocks J. Tidal breath analysis for infant pulmonary function testing. ERS/ATS Task Force on Standards for Infant Respiratory Function Testing. European Respiratory Society/American Thoracic Society. Eur Respir J 2000; 16: 1180-1192.

5. Frey U, Stocks J, Sly P, Bates J. Specification for signal processing and data handling used for infant pulmonary function testing. ERS/ATS Task Force on Standards for Infant Respiratory Function Testing. European Respiratory Society/American Thoracic Society. Eur Respir J 2000; 16: 1016-1022.

6. Sly PD, Tepper R, Henschen M, Gappa M, Stocks J. Tidal forced expirations. ERS/ATS Task Force on Standards for Infant Respiratory Function Testing. European Respiratory Society/American Thoracic Society. Eur Respir J 2000; 16: 741-748.

7. Frey U, Stocks J, Coates A, Sly P, Bates J. Specifications for equipment used for infant pulmonary function testing. ERS/ATS Task Force on Standards for Infant Respiratory Function Testing. European Respiratory Society/ American Thoracic Society. Eur Respir J 2000; 16: 731-740.

8. Stocks J, Sly PD, Morris MG, Frey U. Standards for infant respiratory function testing: what(ever) next? Eur Respir J 2000; 16: 581-584.

9. Godfrey S, Bar-Yishay E, Avital A, Springer C. What is the role of tests of lung function in the management of infants with lung disease? Pediatr Pulmonol 2003; 36: 1-9.

10. Hoo AF, Dezateux C, Hanrahan JP, Cole TJ, Tepper RS, Stocks J. Sex-specific prediction equations for Vmax(FRC) in infancy: a multicenter collaborative study. Am J Respir Crit Care Med 2002, 15; 165: 1084-1092. 
11. Lagerstrand L, Ingemansson M, Bergstrom SE, Lidberg K, Hedlin G. Tidal volume forced expiration in asthmatic infants: reproducibility and reversibility tests. Respiration 2002; 69: 389-396.

12. Turner DJ, Lanteri CJ, LeSouef PN, Sly PD. Improved detection of abnormal respiratory function using forced expiration from raised lung volume in infants with cystic fibrosis. Eur Respir J 1994; 7: 1995-1999.

13. Feher $A$, Castile $R$, Kisling $\mathrm{J}$, et al. Flow limitation in normal infants: a new method for forced expiratory maneuvers from raised lung volumes. J Appl Physiol 1996; 80: 2019-2025.

14. Henschen M, Stocks J, Hoo AF, Dixon P. Analysis of forced expiratory maneuvers from raised lung volumes in preterm infants. J Appl Physiol 1998, 85: 1989-1997.

15. Ranganathan SC, Bush A, Dezateux $C$, et al. Relative ability of full and partial forced expiratory maneuvers to identify diminished airway function in infants with cystic fibrosis. Am J Respir Crit Care Med 2002; 15: 1350-1357.

16. Hülskamp G, Hoo A, Ljungberg H, Lum S, Pillow JJ, Stocks J. Progressive decline in plethysmographic lung volumes in infants: physiology or technology? Am J Respir Crit Care Med 2004 (in press).

17. de Winter JP, Merth IT, Brand R, Quanjer PH. Functional residual capacity and static compliance during the first year in preterm infants treated with surfactant. Am J Perinatol 2000; 17: 377-384.

18. Gerhardt $T$, Hehre $D$, Bancalari $E$, Watson $H$. A simple method for measuring functional residual capacity by $N 2$ washout in small animals and newborn infants. Pediatr Res 1985; 19: 1165-1169.

19. Gustafsson PM, Kallman S, Ljungberg H, Lindblad A. Method for assessment of volume of trapped gas in infants during multiple-breath inert gas washout. Pediatr Pulmonol 2003; 35: 42-49.

20. Gustafsson PM, Aurora P, Lindblad A Evaluation of ventilation maldistribution as an early indicator of lung disease in children with cystic fibrosis. Eur Respir J 2004 (in press).

21. Desager KN, Cauberghs M, Naudts J, van de Woestijne KP. Influence of upper airway shunt on total respiratory impedance in infants. J Appl Physiol 1999; 87: 902-909.

\section{Useful web links}

\section{http://www.ersnet.org/}

The European Respiratory Society's homepage contains useful information on educational and training opportunities, as well as many links and information about the ERS annual congress.

\section{http://www.thoracic.org/}

The American Thoracic Society is an international, educational and scientific society which focuses on respiratory and critical care medicine and hosts the premier international forum for physicians and scientists who work in pulmonary and critical care medicine. The homepage contains useful information on educational and training opportunities, as well as many links.

\section{http://www.ich.ucl.ac.uk/ich/html/ academicunits/portex/index.html}

This site contains a downloadable macro to calculate V'max, FRC Z-scores, as well as information on research and courses and workshops held at the institute of child health in London.

\section{Suggested answers}

1. The methods are whole-body plethysmography and gas dilution tests. The latter can be separated into the closed circuit He dilution test and open circuit washout tests. The open-circuit washout tests can, in turn, be divided into those based on simple bias flow washouts without measurements of tidal volume, and those based on breath-by-breath measurements of expiratory flow, volume and tracer gas concentration.

2. Body plethysmography measures all compressible gas within the thorax, including that not readily in communication with the central airways, whilst the gas dilution test determines the communicating lung volume. The plethysmographic FRC thus includes trapped gas and may be more appropriate for assessing hyperinflation. Some gas dilution tests allow for assessment of ventilation maldistribution, while plethysmography can be used to assess overall airways resistance.

3. The FRC is a more variable static lung volume than the maximum inspired volume after a passive inflation to a given airway pressure. The results from the RVRTC test are therefore thought to be more reproducible and less sensitive to fluctuations in end-expiratory level than the results from the TRTC test. In addition, the RVRTC test resembles adult spirometry to a large extent.

4. Just like in adult spirometry, forced expiratory flows at end-expiration are thought to reflect

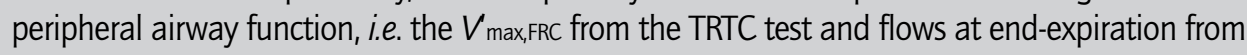
the RVRTC test may provide such information. The difference in FRC measured by plethysmography and that obtained by gas dilution tests may serve as an index of the volume of trapped gas and indirectly peripheral airway obstruction. Finally, obstruction in the peripheral airways results in impaired distribution of ventilation, as measured using a multiple-breath inert gas washout test. 